\title{
Octupole deformation properties of actinide isotopes within a mean field approach
}

\author{
L M Robledo \\ Dep. Física Teórica, Módulo 15, Universidad Autónoma de Madrid, \\ E-28049 Madrid, Spain \\ E-mail: luis.robledo@uam.es \\ R. R. Rodríguez-Guzmán \\ Department of Chemistry, Department of Physics and Astronomy \\ Rice University, Houston, Texas 77005, USA
}

\begin{abstract}
We discuss the octupole deformation properties of many even-even actinide isotopes. The analysis is carried out in the mean field framework (Hartree-Fock-Bogoliubov approximation) by using the axially symmetric octupole moment as a constraint. A one-dimensional octupole collective Hamiltonian is used to obtain properties like excitation energies or E1 and E3 transition probabilities of the negative parity band-heads associated to the lowest lying $1^{-}$and $3^{-}$states. The evolution of these values with neutron number is discussed and a comparison with available experimental data is made. In order to minimize the uncertainties associated to the energy density functional used, the calculations have been carried out for an assorted set ranging from the $\mathrm{BCP} 1$ functional to the finite range Gogny interaction with the D1S, D1N and D1M parametrization.
\end{abstract}

Keywords: Nuclear structure, negative parity octupole states, actinide isotopes

PACS numbers: 21.60.Jz, 21.20.Re

AMS classification scheme numbers: 44

Submitted to: J. Phys. G: Nucl. Phys. 


\section{Introduction}

Nowadays it is well established both theoretical and experimentally that octupole deformation is responsible for some unusual properties of the low energy spectrum of several isotopes of radium and thorium as are the presence of low lying $1^{-}$states in even-even nuclei [1]. As those nuclei are usually quadrupole deformed, the next member of the negative parity rotational band, namely the $3^{-}$is also observed. This $3^{-}$is connected by strong $B\left(E 3,3^{-} \rightarrow 0^{+}\right)$transition probabilities to the ground state. Another typical feature of octupole deformation is the appearance of alternating parity rotational bands. Although there are several examples of parity alternating rotational bands at low spins [1, 2] the parity alternation usually appears at high spin as a consequence of the stabilizing effect of angular momentum on the octupole character of the system [3, 4. (moments of inertia increase with the octupole moment and therefore configurations with higher octupole moments are favored as angular momentum increases).

The appearance of octupole effects is strongly linked to the underlying single particle spectrum [5]. The reason is that octupolarity is enhanced when, in a given major shell, the intruder interacts (via a particle-hole excitation) with a standard parity orbital with three units less of angular momentum (3 $3 \hbar$ is the amount of angular momentum carried out by the octupole operator). This happens between the $j_{15 / 2}$ and $g_{9 / 2}$, the $i_{13 / 2}$ and $f_{7 / 2}$ and the $h_{11 / 2}$ and $d_{5 / 2}$ orbitals. For those regions where both protons and neutrons feel a strong octupole interaction is where octupole related effects are expected. For example, in the region around ${ }_{88}^{224} \mathrm{Ra}$ the proton's Fermi level is close to the $f_{7 / 2}$ orbital that interacts strongly with the empty $i_{13 / 2}$ nearby. Besides, the neutron's Fermi level is near the $g_{9 / 2}$ orbital that strongly interacts through the octupole interaction with the $j_{15 / 2}$ nearby. This combined tendency of both protons and neutrons towards octupole deformation is responsible for the observed features of negative parity states in the region around ${ }^{224} \mathrm{Ra}$ (see [1] for relevant bibliography). An interesting question is whether the octupole deformation effects persists when we increase proton number and move away from the ideal case of Ra. For neutrons we already know the answer as octupole effects are only strong for the radium isotopes with mass numbers in the limited range 222-228 ( $\mathrm{N}=134$ 140). However, neutrons are in a different major shell in the actinides and the dependency with proton number is far from being the same. To answer this question we have carried out a series of calculations in several isotopes of the even-even actinides $\mathrm{U}, \mathrm{Pu}, \mathrm{Cm}, \mathrm{Cf}, \mathrm{Fm}$ and No exploring the possibility of octupole deformed ground states. To this end, we have computed potential energy curves (PECs) as a function of the axially symmetric octupole moment ( $\mathrm{K}=0$ bands) within the constrained Hartree-Fock-Bogoliubov (HFB) approximation [6, 7]. The PECs together with the collective octupole inertia are used as input for a one-dimensional collective Hamiltonian calculation that allows the evaluation of the energy of the lowest lying $1^{-}$ and $3^{-}$states as well as $B(E 1)$ and $B(E 3)$ transition probabilities to the $0^{+}$ground state [8, 9]. Several parametrizations of the Gogny [10, 11] and BCP [12, 13, 14, 15] energy density functionals (EDF) are used. The reason for the consideration of this variety of interactions is its empirical and phenomenological nature. The interactions are fitted to bulk properties of nuclei (binding energies, radii, etc) and not to the spectroscopic quantities considered in this paper. Therefore, a variation of their values with the interaction considered is to be expected. The range of variation with different interactions can be taken as an estimator of the error attributable to the uncertainties 
in the nuclear interaction.

All the nuclei considered in this work have a deep prolate minimum along the quadrupole degree of freedom. As a consequence the coupling between the quadrupole and octupole degrees of freedom is expected to be weak and therefore the octupole degree of freedom alone is going to play a role in the properties of negative parity states [16]. There are other regions of the periodic table [17, 18, 19] where the quadrupoleoctupole coupling as well as triaxiallity effects are relevant.

Recently a theoretical survey of $\mathrm{K}=0$ octupole states for even-even nuclei has been published [20]. In this survey, the Generator Coordinate Method (GCM) with the octupole degree of freedom has been used to compute octupole properties of over 800 even-even nuclei including some of the nuclei discussed in this paper. However, a large fraction of the nuclei studied here are quite close to proton's drip line and were not considered in [20].

\section{Theoretical tools}

The description of octupole properties will be carried out in the mean field framework [6] with the realistic Gogny [10, 11] and BCP [12, 13, 14, 15] EDFs. As pairing correlations play a relevant role in the low energy nuclear dynamics the full HFB approximation will be considered. In this scheme, quasiparticle annihilation and creation operators are defined as linear combinations of a conveniently chosen single particle basis. In our case, we have used a Harmonic Oscillator (HO) basis which has been chosen big enough as to warrant convergence of the physical quantities (energies, transition probabilities, etc) with the basis size. To solve the constrained HFB equations an approximate second order gradient method [21], based on the parametrization of the energy in terms of the Thouless expansion of the most general HFB wave functions and using two quasiparticle energies as precondititioner, has been used. Axial symmetry has been preserved in the calculation suggesting the use of an axially symmetric $\mathrm{HO}$ basis made up of the tensor product of two dimensional $\mathrm{HO}$ wave functions times one-dimensional $\mathrm{HO}$ ones. Along with the octupole moment constraint associated to the multipole operator $\hat{Q}_{3}=r^{3} Y_{30}$ and used to generate the potential energy curves (PEC's), we have included a constraint on the center of mass of the nucleus (i.e. the mean value of $r^{1} Y_{10}$ has been set to zero) to prevent the coupling to the spurious center of mass motion. As a consequence of the axial symmetry imposed in the HFB wave functions the remaining components $\mu \neq 0$ of the corresponding multipole operators $r^{3} Y_{3 \mu}$ and $r Y_{1 \mu}$ have a zero mean value by construction.

The information given by mean field theories is restricted to the energy and shape of the -generally- deformed ground state. To restore the parity symmetry broken by the solutions of the mean field approximation and in order to describe the dynamics of the collective excited states of octupole character it is mandatory to go beyond the mean field approximation. With this in mind, the octupole degree of freedom $Q_{3}=\left\langle\psi\left|\hat{Q}_{3}\right| \psi\right\rangle$ (where $|\psi\rangle$ is the HFB intrinsic wave function) has been used to build up a collective Hamiltonian based on the GCM and the Gaussian Overlap Approximation (GOA) [22, 23, 24]. In this method, the GOA is used to reduce the Hill-Wheeler equation of the GCM to a Schrödinger equation for the collective wave function, the so-called Collective Schrödinger Equation (CSE)

$$
\hat{\mathcal{H}}_{\text {coll }} \phi_{\alpha}\left(Q_{3}\right)=\epsilon_{\alpha} \phi_{\alpha}\left(Q_{3}\right)
$$


where the collective Hamiltonian $\hat{\mathcal{H}}_{\text {coll }}$ is given by

$$
\begin{aligned}
\hat{\mathcal{H}}_{\text {coll }} & =-\frac{1}{\sqrt{G\left(Q_{3}\right)}} \frac{\partial}{\partial Q_{3}} \sqrt{G\left(Q_{3}\right)} \frac{1}{2 B\left(Q_{3}\right)} \frac{\partial}{\partial Q_{3}} \\
& +V\left(Q_{3}\right)-\epsilon_{0}\left(Q_{3}\right) .
\end{aligned}
$$

In this expression $G\left(Q_{3}\right)$ is the metric, $B\left(Q_{3}\right)$ is the mass parameter associated with the collective motion along $Q_{3}, V\left(Q_{3}\right)$ is the collective potential given by the HFB energy $V\left(Q_{3}\right)=\left\langle\psi\left(Q_{3}\right)|\hat{H}| \psi\left(Q_{3}\right)\right\rangle$ and $\epsilon_{0}\left(Q_{3}\right)$ is the Zero Point Energy (ZPE) correction. The eigenfunctions $\phi_{\alpha}\left(Q_{3}\right)$ of equation (11) have to be normalized to one with the metric $G\left(Q_{3}\right)$

$$
\int d Q_{3} \sqrt{G\left(Q_{3}\right)} \phi_{\alpha}^{*}\left(Q_{3}\right) \phi_{\beta}\left(Q_{3}\right)=\delta_{\alpha, \beta}
$$

in order to preserve the hermiticity of $\hat{\mathcal{H}}_{\text {coll }}$.

It has to be mentioned that a collective Schrödinger equation can also be obtained from the Adiabatic Time Dependent Hartree-Fock (ATDHF) theory [25, 26, 27, after quantization of the semi-classical Hamiltonian for the slow moving collective degrees of freedom. The collective Hamiltonian obtained in this way has the same functional form as the GCM+GOA one, but the expression of the collective parameters is different. Later on we will discuss how to choose these collective parameters.

An interesting property of $\hat{\mathcal{H}}_{\text {coll }}$ is its invariance under the exchange $Q_{3} \rightarrow-Q_{3}$ that allows the classification of its eigenfunctions, $\phi_{\alpha}\left(Q_{3}\right)$, according to their parity under the $Q_{3} \rightarrow-Q_{3}$ exchange. It is easy to see that the parity of the collective wave function under the $Q_{3} \rightarrow-Q_{3}$ exchange corresponds to the spatial parity operation in the correlated wave function built up from $\phi_{\alpha}$. The inclusion of octupole correlations immediately restores the parity symmetry lost at the mean field level [8, 28, Therefore, the solution of the CSE equation (11) allows the calculation of the $0^{+}-1^{-}\left(3^{-}\right)$energy splitting as well as the $B(E 1)$ and $B(E 3)$ transition probabilities connecting them. At this point it has to be pointed out that in the present framework where only time reversal invariant wave functions are considered it is only possible to describe excited states with average angular momentum zero. To deal with genuine $1^{-}$or $3^{-}$states a projection onto good angular momentum should be performed, which is out of the scope of the present work. Here we will assume that the cranking rotational energy of the $1^{-}$state is much smaller than the excitation energy of the negative parity band head and therefore can be safely disregarded. With this approximation in mind, the reduced transition probabilities from the lowest $1^{-}$and $3^{-}$states to the $0^{+}$ground state can be computed within the Rotational Model approximation as

$$
B\left(E \lambda, I_{f} \rightarrow I_{i}\right)=e^{2}\left\langle I_{i} K \lambda 0 \mid I_{f} K\right\rangle^{2}\left|\left\langle\varphi_{i}\left|r^{\lambda} Y_{\lambda, 0}\right| \varphi_{f}\right\rangle\right|^{2},
$$

where $\left|\varphi_{i}\right\rangle$ and $\left|\varphi_{f}\right\rangle$ are correlated wave functions obtained in the spirit of the GCM from the collective wave functions $\phi_{\alpha}\left(Q_{3}\right)$. The above formula can be reduced to an expression involving those collective wave functions $\phi_{\alpha}\left(Q_{3}\right)$ by means of the GOA [30]. The final result for $K=0$ bands reads

$$
B\left(E 1,1^{-} \rightarrow 0^{+}\right)=\frac{e^{2}}{4 \pi}\left|\left\langle\phi_{0^{-}}\left|D_{0}\right| \phi_{0^{+}}\right\rangle_{\mathrm{COLL}}\right|^{2}
$$

for the E1 electric transition and

$$
B\left(E 3,3^{-} \rightarrow 0^{+}\right)=\frac{e^{2}}{4 \pi}\left|\left\langle\phi_{0^{-}}\left|Q_{30}(\mathrm{PROT})\right| \phi_{0^{+}}\right\rangle_{\mathrm{COLL}}\right|^{2},
$$


for the E3 one. In the above formulas we have introduced the collective matrix element of an operator $\hat{O}$ as

$$
\left\langle\phi_{0^{-}}|\hat{O}| \phi_{0^{+}}\right\rangle_{\mathrm{COLL}}=\int d Q_{3} G^{1 / 2} \phi_{0^{-}}^{*}\left(Q_{3}\right) O\left(Q_{3}\right) \phi_{0^{+}}\left(Q_{3}\right)
$$

where $O\left(Q_{3}\right)=\left\langle\psi\left(Q_{3}\right)|\hat{O}| \psi\left(Q_{3}\right)\right\rangle$. In the formula (6) $D_{0}$ is the dipole moment operator whose mean value is defined as the difference between the center of mass of protons and neutrons

$$
D_{0}\left(Q_{3}\right)=\frac{N}{A}\left\langle\psi\left(Q_{3}\right)\left|\hat{z}_{\text {prot }}\right| \psi\left(Q_{3}\right)\right\rangle-\frac{Z}{A}\left\langle\psi\left(Q_{3}\right)\left|\hat{z}_{\text {neut }}\right| \psi\left(Q_{3}\right)\right\rangle .
$$

Finally, $Q_{30}(\mathrm{PROT})$ is the part of the octupole operator acting on proton's space. In the recent survey of 20 it was pointed out the inadequacy of the rotational formula for the calculation of E1 and E3 transition probabilities for near spherical nuclei (see [29] for a beautiful example of how angular momentum projection solves a related problem near ${ }^{208} \mathrm{~Pb}$ ). This is not a problem in the present calculation as the nuclei considered are well deformed and the rotational formula works well there.

To carry out the collective calculations it is necessary to specify the collective parameters $G\left(Q_{3}\right), B\left(Q_{3}\right)$ and $\epsilon_{0}\left(Q_{3}\right)$ appearing in the definition of $\hat{\mathcal{H}}_{\text {coll }}$ equation (22). As it was mentioned before, there are two sets of parameters coming from the $\mathrm{GCM}+\mathrm{GOA}$ and the ATDHF derivation of the collective Hamiltonian. The set of parameters used in this calculation is an admixture of the two and it is known as the ATDHF $+\mathrm{ZPE}$ set. It includes the mass parameter $B\left(Q_{3}\right)$ coming out from the semi-classical Hamiltonian of the ATDHF theory, the metric of the GCM+GOA and the ZPE correction computed with the GCM+GOA formula but using the ATDHF mass instead, i.e.

$$
\epsilon_{0}\left(Q_{3}\right)=\frac{1}{2} G\left(Q_{3}\right) B\left(Q_{3}\right)_{A T D H F}^{-1} .
$$

This set of parameters was devised to put together the advantages of the ATDHF set (time-odd components included in the mass term) and the ones of the GCM+GOA (ZPE correction). This method can be somewhat justified in the context of the extended Generator Coordinate method [24, 31] and has been extensively used [11, 8]. In mean field calculations it is customary to include in the ZPE correction the rotational energy. We have tested that the inclusion of the rotational energy correction (as computed in [36] to take into account effects near sphericity and the approximate calculation of the Yoccoz moment of inertia) does not modify substantially the results, as could be anticipated due to the weak coupling of the octupole and quadrupole degrees of freedom.

The calculation of the collective parameters involves the inversion of the HFB stability matrix which is closely related to the matrix of the RPA equation. At present, this is a formidable task and approximations are needed. The approximation used in this paper - called "cranking approximation" [32, 33] - neglects the off-diagonal terms of the stability matrix allowing to invert it analytically but at the cost of including the two body interaction only through the mean field. Although this approximation has been extensively used in the literature for the calculation of collective masses and moments of inertia (see for instance [34, 11, 35]) its validity has not been properly established. Using the "cranking approximation", the ATDHF+ZPE parameters are given by

$$
G\left(Q_{3}\right)=\frac{M_{-2}\left(Q_{3}\right)}{2 M_{-1}^{2}\left(Q_{3}\right)}, B\left(Q_{3}\right)=\frac{M_{-3}\left(Q_{3}\right)}{M_{-1}^{2}\left(Q_{3}\right)}
$$


where the quantities $M_{-n}\left(Q_{3}\right)(n=1,2,3)$ are defined as

$$
M_{-n}\left(Q_{3}\right)=\sum_{k, l} \frac{\left|\left(Q_{30}\right)_{k l}^{20}\right|^{2}}{\left(E_{k}+E_{l}\right)^{n}} .
$$

In the above expression, $E_{k}$ are the quasi-particle energies and $\left(Q_{30}\right)_{k l}^{20}$ are the matrix elements of the 20 part [6] of the octupole operator $\hat{Q}_{30}$ in the quasi-particle basis of the HFB wave function $\left|\psi\left(Q_{3}\right)\right\rangle$. This form of the collective mass is usually referred to in the literature as the Belyaev-Inglis inertia [6].

\subsection{Interactions}

In this paper we have used the three modern parametrizations of the Gogny functional [10] which are available in the market, namely Gogny D1S [11], D1N [37] and D1M [38]. There are mainly two reasons to proceed in this way. First, to gain confidence on the independence of our results concerning tiny details of the interaction/parametrization used. In this respect, we have to comment that although the three Gogny's parametrizations used belong to the same functional form of the interaction, their different behavior regarding nuclear matter, pairing properties, binding energies or radii suggest their non-equivalence. The other reason to do the calculations with the D1N and D1M parametrizations is to check their performance in describing octupole deformation phenomena as a partial test to asses their ability to describe low energy nuclear phenomena (see for instance [39, 40] for recent studies with D1M). In addition to the Gogny force, we have used the recently proposed BCP [12] energy density functional with the BCP1 parameter set [12]. This functional is based on a local density approximation to a realistic nuclear matter equation of state supplemented by a finite range surface term. The spin-orbit and Coulomb interactions have the standard form as used in the Gogny force. The pairing force used is a zero range, density dependent force that was fitted to reproduce the pairing gap in nuclear matter given by the Gogny functional. The parameters have been fitted to reproduce binding energies and radii of selected spherical nuclei and the results show a reasonable level of agreement comparable or even better than the results provided by D1S. Several spectroscopic quantities have been computed in several regions of the periodic table and the results are in close agreement with the experimental values and the theoretical results of D1S [13, 15]. Octupole properties of $\mathrm{Ra}$, Th and $\mathrm{Ba}$ isotopes have been analyzed in the past with this functional [14] and the good agreement obtained at a qualitative level in the comparison with the experiment and Gogny force calculations is a clear indication of the suitability of this EDF for the exploration of the octupole degree of freedom in the heavy actinides.

\section{Results}

In the eight isotopic chains considered we have followed the same procedure, first the octupole constrained HFB wave functions have been generated along the lines described in the previous section. Next the collective mass and zero point energies are computed with the mean field wave functions and together with the PEC itself they are used to build the Collective Schrödinger Hamiltonian. From the lowest lying solutions of the CSE we obtain the excitation energy of the first negative parity excited state (a $1^{-}$for these deformed nuclei) and the $B\left(E 1,1^{-} \longrightarrow 0^{+}\right)$and $B\left(E 3,3^{-} \longrightarrow 0^{+}\right.$) 


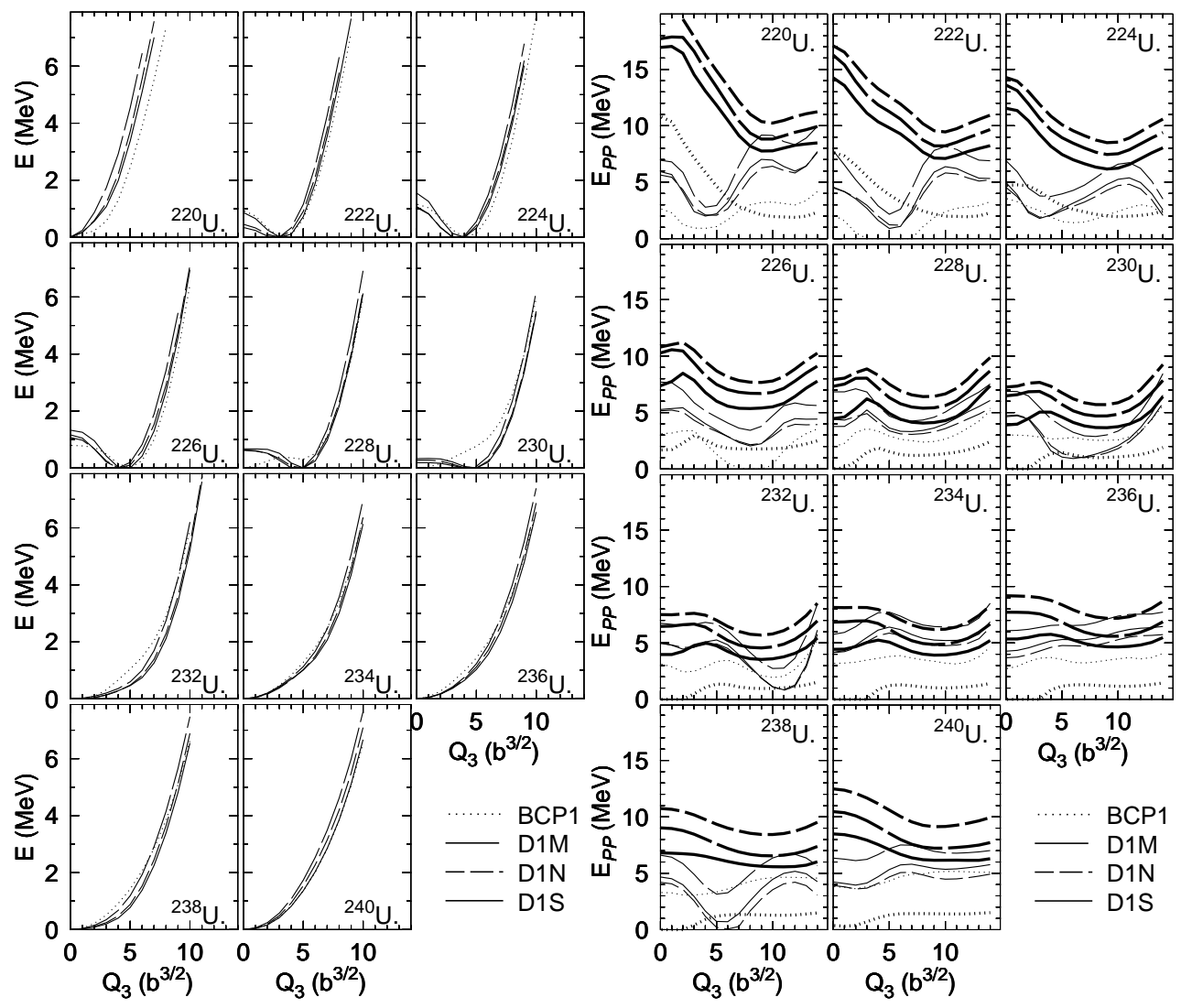

Figure 1. On the left hand side panels, the PEC's as a function of the octupole moment $Q_{3}$ (in thousands of Fermi cube or $b^{3 / 2}$ ) for the even-even isotopes of uranium with $A$ in the range between $A=220$ and $A=240$. Results for the D1S (full lines), D1N (dashed lines), D1M (dashed dotted lines) parametrizations of the Gogny and the BCP1 (dotted lines) functionals are presented. On the right hand side panels the particle-particle correlations energies for both protons (thick curves) and neutrons (thin curves) are given as a function of the octupole moment in $b^{3 / 2}=10^{3} \mathrm{fm}^{3}$

transition probabilities (the $3^{-}$state in the $B(E 3)$ transition probability refers to the second member of the rotational band built on top of the $1^{-}$band head). The whole procedure will be thoroughly described for the case of the uranium isotope. For the other isotopic chains only the most relevant results, namely the PES and the observables obtained after solving the CSE will be presented.

\subsection{Low excitation energy properties of the uranium isotopes}

In fiugre 1 we display, as a function of the octupole moment $Q_{3}$ and the four interactions used, the PECs (left hand side panels) and the particle-particle correlation energies (right hand side panels). The particle-particle correlation energy is defined as $E_{p p}=\frac{1}{2} \operatorname{Tr} \Delta \kappa$, and it is given in terms of the pairing field $\Delta$ and the pairing tensor $\kappa$. This quantity gives a rough idea of the amount of pairing correlations in the system. It can also be used as an indicator of where the single particle level 
density around the Fermi surface is high or low as high level densities are associated to strong pairing correlations. This quantity is also correlated with the pairing gap that represents the energy of the lowest two quasiparticle excitation and therefore it is closely related to the collective inertias to be discussed below. Coming back to the figure, the PECs obtained with the four interactions considered are very similar. For the particle-particle correlation energies the same similarity is obtained for the three parametrization of Gogny, but for the BCP functional the particle-particle correlation energies are significantly smaller. Of the eleven isotopes considered, five $\left({ }^{222-230} \mathrm{U}\right)$ show an octupole deformed minimum with a depth never exceeding the $1.5 \mathrm{MeV}$. The octupole moment of the minima changes with the neutron number in a non-trivial way. The remaining nuclei with a minimum at $Q_{3}=0$ show a parabolic behavior centered around the minimum with a curvature that is more or less independent of the interaction considered. There seems to be a certain correlation between the appearance of octupole deformed minima and the minima of the neutron pairing energies of the right hand side panels. This is something to be expected as the presence of deformed minima is usually related (Jahn-Teller effect) to regions of low level density of the underlying single particle orbitals and these low level density regions usually correspond to small pairing correlations.

As the octupole moment increases, the nuclear shape changes and the values of other multipole moments including the quadrupole, hexadecapole and dipole moments change. In the left hand side panels of figure 2 the $\beta_{2}$ and $\beta_{4}$ deformation parameters are represented as a function of $Q_{3}$. For most of the $\mathrm{U}$ isotopes considered the variation with $Q_{3}$ is small and it is only in the lightest nuclei ${ }^{220} \mathrm{U}$ and ${ }^{222} \mathrm{U}$ where the changes are noticeable. The nucleus ${ }^{220} \mathrm{U}$ is spherical at $Q_{3}=0$ and develops a sizable quadrupole deformation with increasing octupole moment. On the left hand side panels of this figure the dipole moment of equation (8) is plotted. The dipole moment is zero by construction at $Q_{3}=0$ and its behavior with the octupole moment is determined by the occupancies of specific single particle orbitals around the Fermi level. High $K$ orbitals give the largest contributions to $D_{0}$ and therefore, depending on their occupancy for protons or neutrons as a function of $Q_{3}$, we can observe different behaviors. For the lightest isotopes the dipole moment increases almost linearly with $Q_{3}$. The slope decreases with neutron number to the point that for ${ }^{236} \mathrm{U}$ the value of $D_{0}$ is almost zero for a wide range of octupole moments as a consequence of competing neutron and protons contributions. Given the intimate connection between the dipole moment and the $B(E 1)$ transition probabilities we conclude that the situation found in ${ }^{236} \mathrm{U}$ will lead to a quenched $B(E 1)$ value as compared to the ones of other isotopes of the same nucleus.

In figure 3 the collective inertia $B\left(Q_{3}\right)$ of equation (10) is depicted as a function of $Q_{3}$. It plays a central role in the outcome of the collective Schrödinger Hamiltonian calculations mentioned in the previous section. The collective inertia is roughly speaking inversely proportional to the pairing correlations (the pairing gap to be more specific) and therefore the similarity between the collective masses obtained for D1S, D1N and D1M indicate that a subtle cancellation between proton's and neutron's contributions has to take place (see the particle-particle correlation energies in figure 1). It is also worth to notice that the peaks observed in the $B\left(Q_{3}\right)$ plots are related to regions of low pairing correlations. On the other hand, the $\mathrm{BCP} 1$ inertia is systematically higher than the ones of the different parametrizations of the Gogny functional. The difference amounts to factors of two or even more in some specific cases. This will be responsible for the differences observed in the solution of the CSE. 

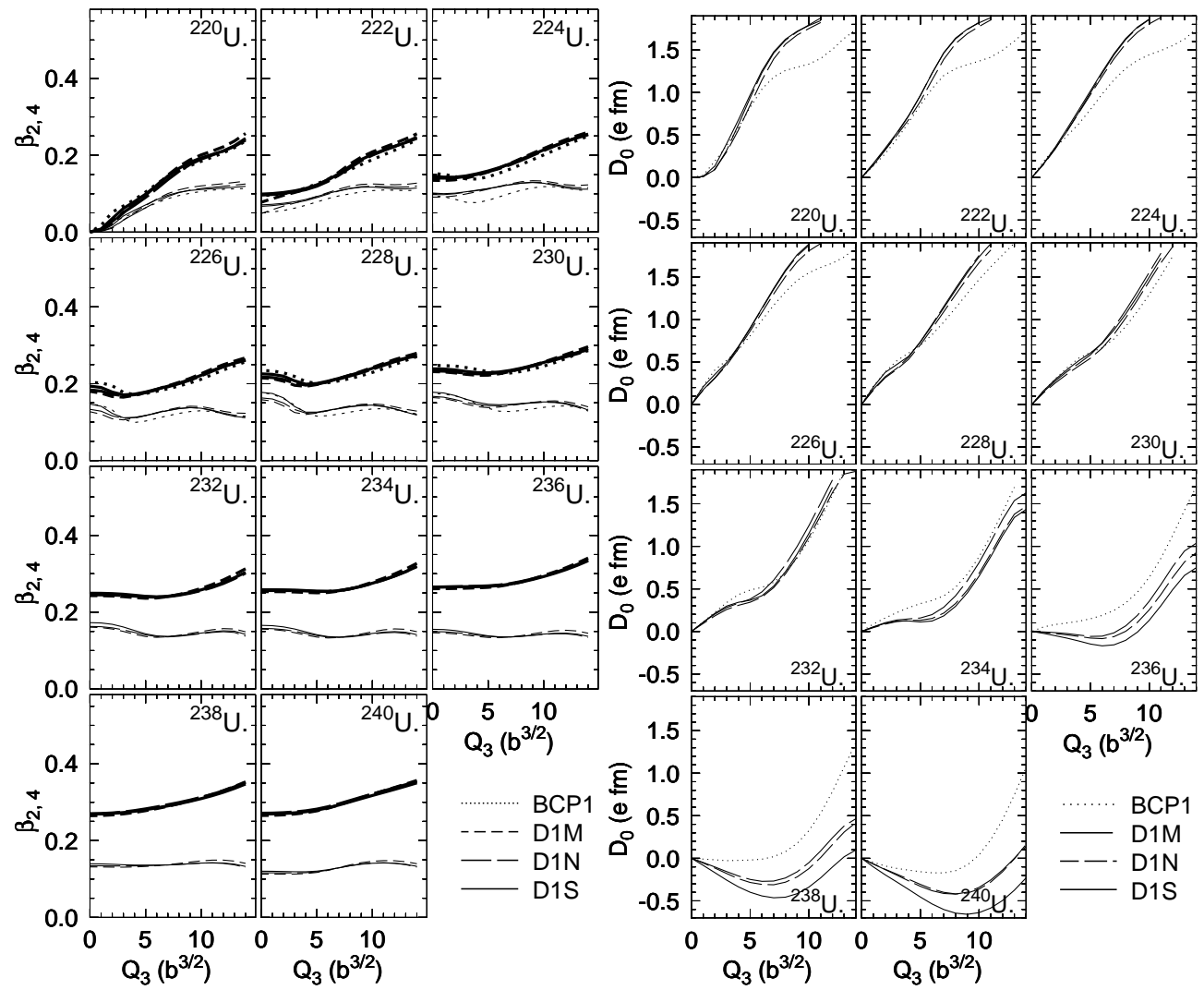

Figure 2. On the left hand side panels, the $\beta_{2}$ (thick lines) and $\beta_{4}$ (thin lines) deformation parameters are plotted as a function of the octupole moment $Q_{3}$ (in $b^{3 / 2} \equiv 10^{3} \mathrm{fm}^{3}$ ) for the even-even isotopes of uranium with $A$ in the range between $A=220$ and $A=240$. Results are given for the D1S (full lines), D1N (dashed lines), D1M (dashed dotted lines) and BCP1 (dotted lines) functionals. On the right hand side panels the dipole moment is shown as a function of $Q_{3}$.

On the right hand side panels of figure 3 the zero point energy correction $\epsilon\left(Q_{3}\right)$ of equation (9) is depicted for the isotopes of uranium. The $\epsilon\left(Q_{3}\right)$ values are correlated with the inverse of the collective inertia $B\left(Q_{3}\right)$ as can easily be noticed in the figure and its behavior with $Q_{3}$ can modify somehow the topology of the PES but not at the level of changing significantly the physical results. On the average the $\epsilon\left(Q_{3}\right)$ values are of the order of $1.5 \mathrm{MeV}$ which is consistent with the correlation energies for parity symmetry restoration and octupole dynamics obtained in [20]. This energy together with the energy gain obtained by the breaking of reflection symmetry at the mean field level (of the order of one $\mathrm{MeV}$ at most) should be added to the nucleus binding energy but the relatively constant values as a function of mass number indicate that the impact of such addition in separation energies is not expected to be significant. After solving the one-dimensional collective Schrödinger equation we obtain the excitation energies of the $1^{-}$states and the $B\left(E 1,1^{-} \rightarrow 0^{+}\right)$and $B\left(E 3,3^{-} \rightarrow 0^{+}\right)$transition probabilities. The results obtained with Gogny D1S, D1N and D1M as well as for the $\mathrm{BCP} 1$ energy density functional for the $\mathrm{U}$ isotopes are depicted along with available experimental values 41]in the left hand side panels of figure 4. For all the results 


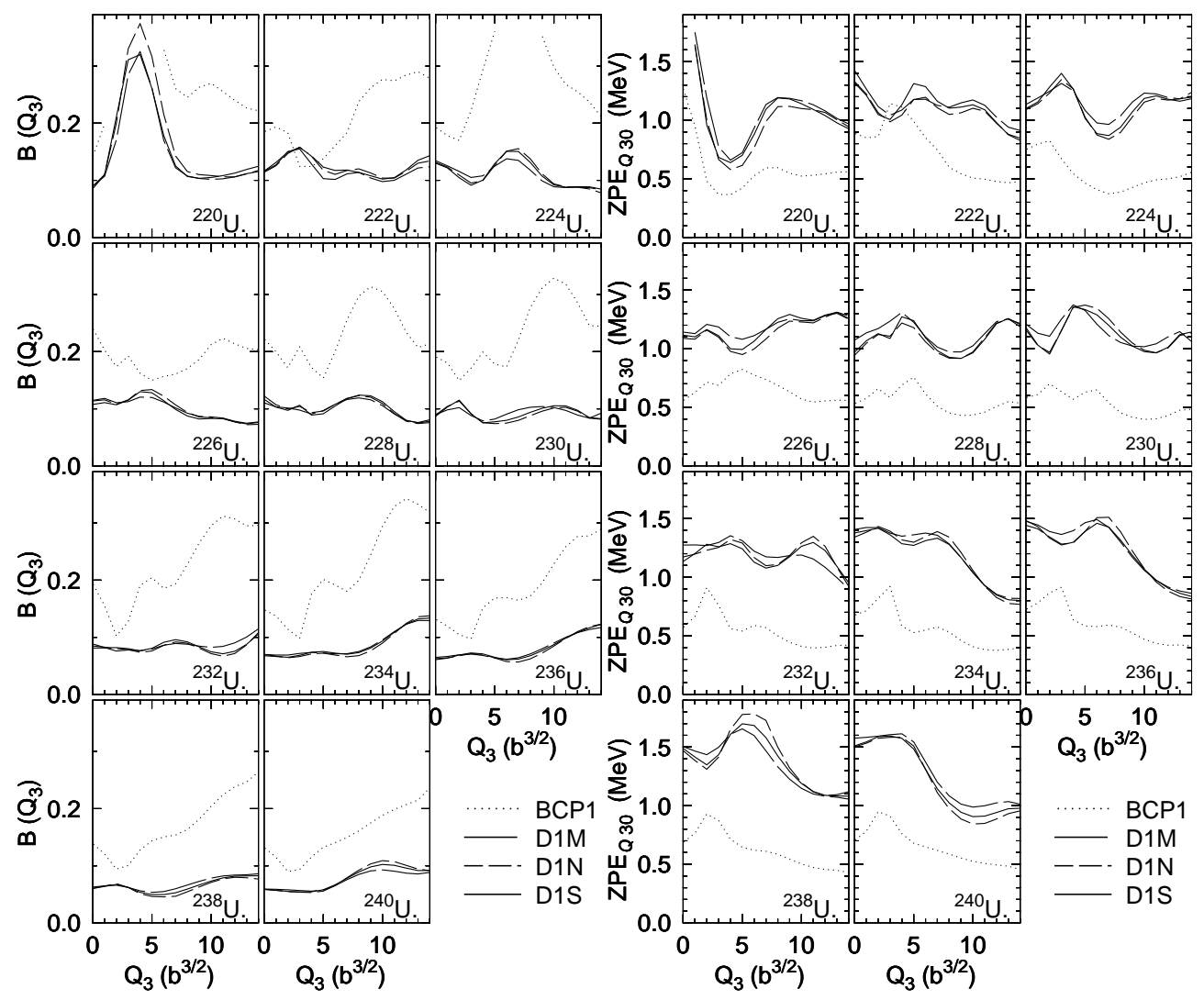

Figure 3. On the left hand side panels the octupole collective inertia parameter $B\left(Q_{3}\right)$, which is one of the relevant ingredients in the one-dimensional collective Schrödinger Hamiltonian (see text for details), is shown as a function of $Q_{3}$ for the considered $\mathrm{U}$ isotopes. On the right hand side panels the zero point energy (ZPE) correction of equation (9) is represented as a function of $Q_{3}$.

considered we observe that the three parametrizations of the Gogny EDF produce the same isotopic trend being the differences not very relevant from a physical point of view. Obviously, the differences are larger for the most sensitive quantities, namely the transition probabilities. The $1^{-}$excitation energies are very small for the ${ }^{222} \mathrm{U}$ to ${ }^{230} \mathrm{U}$ isotopes. This is the expected behavior as those nuclei show permanent octupole deformation at the HFB level (see figure 11). For the other U isotopes, the HFB energy shows a minimum at $Q_{3}=0$ with a parabolic behavior characteristic of octupole vibrational states which lie higher in energy, as observed in the energies of the negative parity excited states of figure 4 . The comparison with experimental data is reasonable as we are able to reproduce the increasing octupolarity as neutron number decreases. Concerning the $B\left(E 1,1^{-} \rightarrow 0^{+}\right)$transition probabilities we observe a pronounced minimum around $A=236$ that is a direct consequence of the behavior of the dipole moment with $Q_{3}$ observed in figure 2. Unfortunately, there is no experimental data available for these isotopes concerning the $B(E 1)$ transition probabilities and the associated dipole moments [42. The $B\left(E 3,3^{-} \rightarrow 0^{+}\right)$transition probabilities show a marked maximum around $A=226$ that is correlated to the minimum in the energies 
of the $1^{-}$states. For the heavier isotopes the computed $B(E 3)$ compare well with experimental data. On the other hand, the BCP1 results compare reasonably well with the Gogny ones except for the ${ }^{228-232} \mathrm{U}$ isotopes where the octupole correlations are weaker and therefore the $1^{-}$excitation energies are higher and the $B(E 3)$ transition probabilities lower. Also the minimum observed in the $B(E 1)$ as a function of neutron number is shifted two units of neutron number when compared with the Gogny results. In the right hand side panels, a comparison is made between the theoretical results obtained in the present framework and the ones of the GCM with the octupole degree of freedom as generating coordinate (GCM-Q3) obtained for Gogny D1S in [20]. In both calculations the underlying mean field is the same and therefore it should not be surprising the agreement between the results of both calculations. The differences observed in the excitation energies and $B(E 3)$ transition probabilities can be attributed to the different collective masses used in each of the calculations $\mathbb{G}$. However, the magnitude of the differences is compatible with the magnitude of the differences attributed to the use of different parametrizations and/or functionals in the calculations. The message that the comparison of all these theoretical predictions is telling us is that the behavior of the physical quantities as a function of mass number is independent of the kind of calculation and interaction and therefore can be taken as a strong prediction. On the other hand, the values obtained for the physical quantities oscillate typically in a range of plus or minus $40 \%$ and therefore their values should be taken just as an indication of the order of magnitude to be expected.

\subsection{Plutonium isotopes}

Results for the Plutonium ( $\mathrm{Z}=94)$ isotopes in the mass range 222-242 (corresponding to neutron numbers $128-148$ ) are discussed in this section. For this and the remaining isotopic chains to be discussed, we will focus on physical quantities like excitation energies and transition probabilities and the only "intrinsic" quantity to be shown is the PES as a function of the octupole moment to quantify the gain in binding energy associated to octupole correlations. In figure 5 we have plotted on the left hand side the PES as a function of the octupole moment for the $\mathrm{Pu}$ isotopes considered. Well deformed octupole minima are obtained for the isotopes ${ }^{224} \mathrm{Pu},{ }^{226} \mathrm{Pu}$ and ${ }^{228} \mathrm{Pu}$ with mean field octupole correlation energies as large as $1 \mathrm{MeV}$ in ${ }^{226} \mathrm{Pu}$. The nuclei ${ }^{230-234} \mathrm{Pu}$ show very shallow minima which are located at $Q_{30}=0$ in the heaviest of the three. For the other isotopes, the minima are reflection symmetric $\left(Q_{30}=0\right)$ showing a parabolic behavior as a function of $Q_{30}$ with a rather large curvature. On the right hand side the $1^{-}$excitation energy as well as the $B(E 1)$ and $B(E 3)$ transition probabilities (in Weisskopf units) are plotted as a function of mass number. We observe that a range of isotopes starting with ${ }^{224} \mathrm{Pu}$ and ending with ${ }^{232} \mathrm{Pu}$ show octupole deformation in their ground state. The mean field octupole correlation energy is of the order of $1 \mathrm{MeV}$ in the lighter isotopes and gets reduced to a few hundred $\mathrm{keV}$ for ${ }^{232} \mathrm{Pu}$. As a consequence, the $1^{-}$excitation energy for those five isotopes is of the order of $0.5 \mathrm{MeV}$ and much smaller than in the neighboring isotopes. As a result of the enhanced octupolarity in these isotopes the $B(E 3)$ transition probabilities are stronger reaching values of up to $60 \mathrm{~W} . u$. The $B(E 1)$ transition probabilities show a rather constant behavior for the octupole deformed isotopes and show a dip for ${ }^{238} \mathrm{Pu}$

$\ddagger$ In the GCM-Q3 calculation there is no explicit collective mass as the full Hill-Wheeler equation is solved but, if a local approximation to the non-local HW equation is considered the concept of collective mass can be recovered, see Ref [6] for a discussion. 

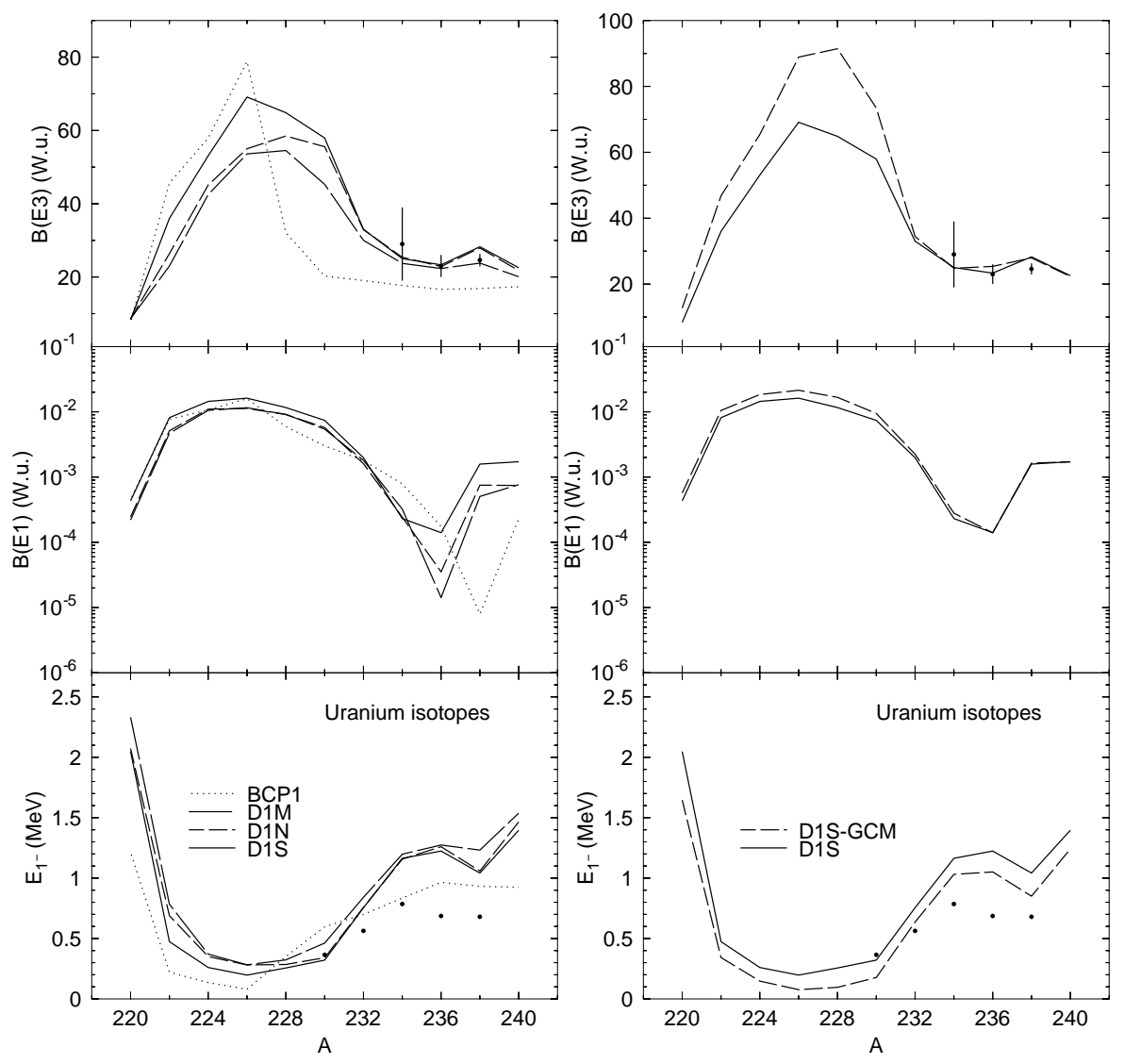

Figure 4. Left hand side panels: Results of the 1D collective Schrödinger Hamiltonian calculations for the three parametrizations of Gogny (D1S, D1N and $\mathrm{D} 1 \mathrm{M})$ as well as for the BCP1 energy density functional. In the lowest panel the energies of the $1^{-}$states as a function of the mass number for the uranium isotopes are depicted. Theoretical results (lines) are plotted along with experimental data (dots). In the middle panel the $B\left(E 1,1^{-} \rightarrow 0^{+}\right)$transition probabilities in W.u. are given. Finally, in the upper panel the $B\left(E 3,3^{-} \rightarrow 0\right)$ are also given, for the different isotopes and different theoretical prediction, in W.u. In the right hand side panels a comparison is made between the results obtained in the present approach and the ones obtained with Gogny D1S in the framework of the GCM for the octupole degree of freedom.

in the case of D1S and ${ }^{240} \mathrm{Pu}$ in the case of both D1N and D1M.

\subsection{Curium results}

Results for the Curium ( $\mathrm{Z}=96)$ isotopes with neutron numbers in between $\mathrm{N}=126$ and $\mathrm{N}=146$ are discussed in this section. In figure 6 we have plotted on the left hand side the PES as a function of the octupole moment. Octupole deformed minima are obtained for the nuclei ${ }^{226-230} \mathrm{Cm}$ with energy gains of the order of a few hundreds of $\mathrm{keV}$ being ${ }^{226} \mathrm{Cm}$ the nucleus with the deepest octupole deformed well. For ${ }^{232-234} \mathrm{Cm}$ the minima are at $Q_{30}=0$ but the minima are in the two cases rather shallow. For the other nuclei the minima is at $Q_{30}=0$ and the PES shows a parabolic behavior with a 

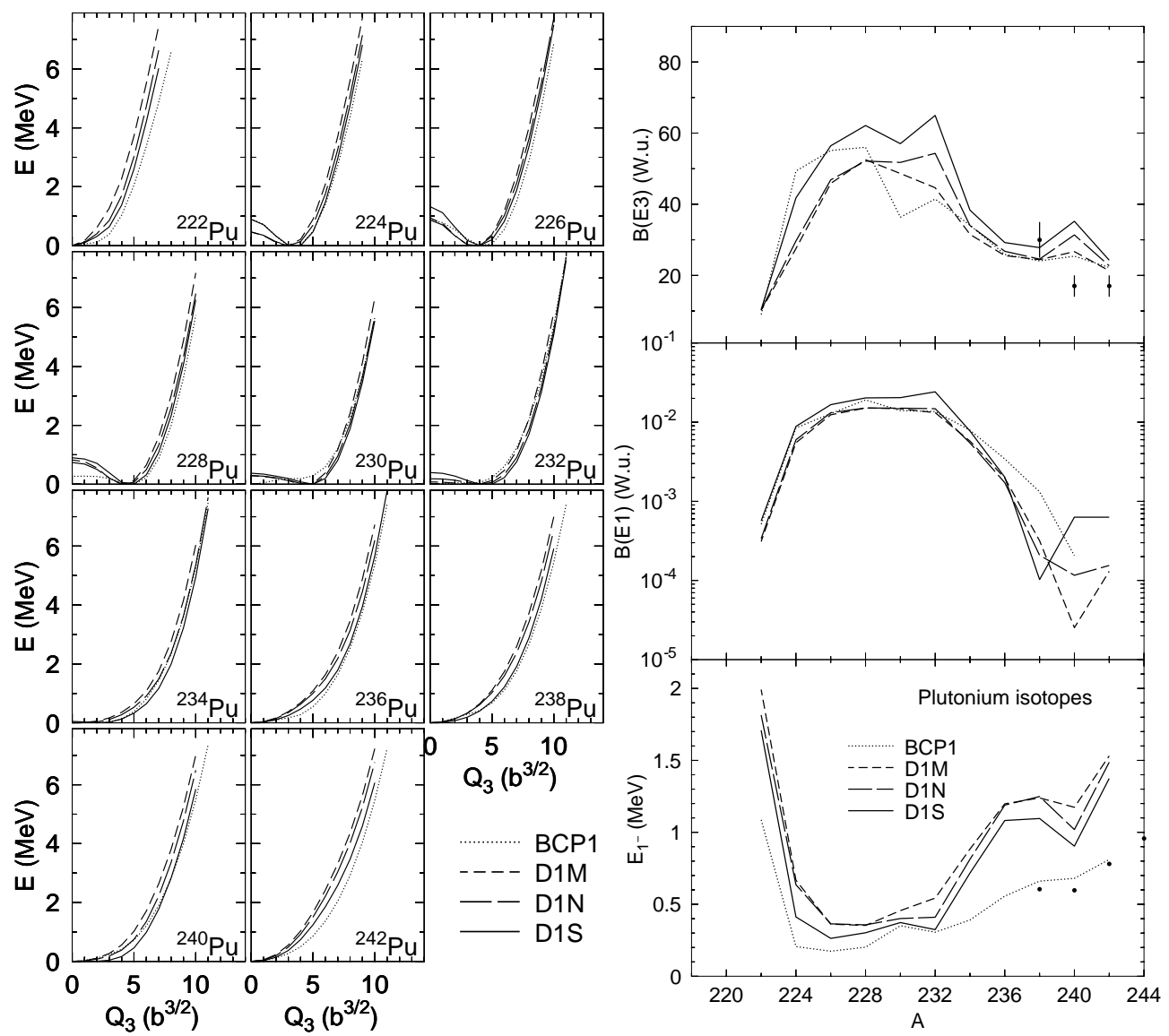

Figure 5. On the left hand side panels, the PES, as a function of $Q_{3}$, are shown for the considered $\mathrm{Pu}$ isotopes. In the right hand side panels, the $1^{-}$excitation energies (in $\mathrm{MeV}$ ) as well as the $B(E 1)$ and $B(E 3)$ transition probabilities (in W.u.) are plotted as a function of the mass number $A$. Theoretical results are depicted as lines of various types (see legends) and the experimental data, taken from 41, are represented by bullets.

rather large curvature. On the right hand side panels, the $1^{-}$excitation energy as well as the $B(E 1)$ and $B(E 3)$ transition probabilities (in Weisskopf units) obtained after solving the 1D CSE are plotted as a function of mass number. The results resemble at a qualitative level the ones for the $\mathrm{Pu}$ isotopes indicating the relatively low impact on octupole correlations of the addition of two protons. Strong octupole correlations, with low lying $1^{-}$states are anticipated to show up in the mass range 226-232. In that range the $B(E 3)$ transition probabilities are rather collective and are around $50 \mathrm{~W} . u$. For the nucleus ${ }^{234} \mathrm{Cm}$ the results show a dependency with the interaction, pointing to a characterization of this nucleus as transitional in terms of octupole correlations.

\subsection{Californium}

Results for the Californium ( $\mathrm{Z}=98)$ isotopes with neutron numbers in the $124-144$ range are presented and discussed in this section. In figure 7 we have plotted on the 

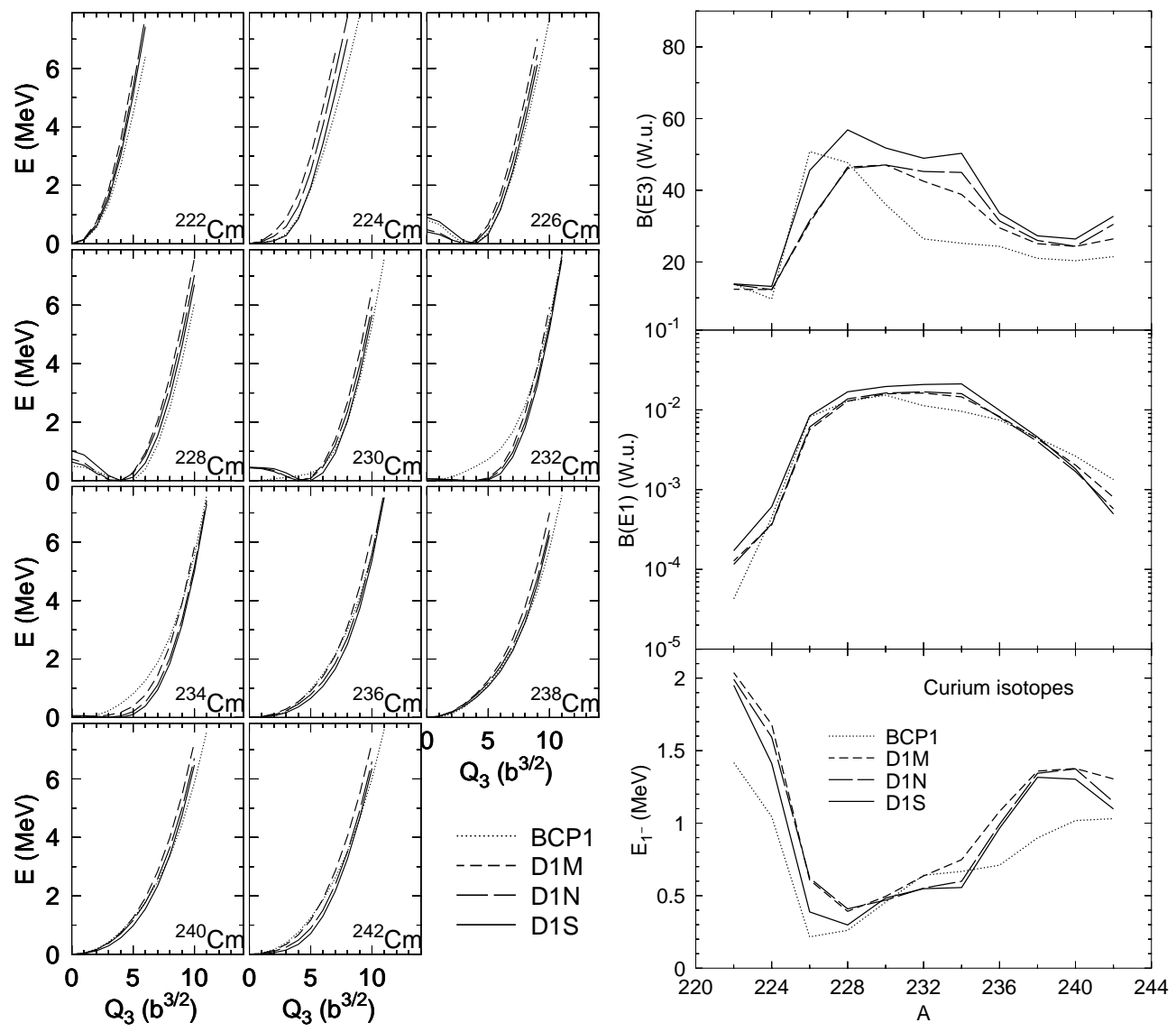

Figure 6. Same as figure 5 but for the curium isotopes

left hand side the PES as a function of the octupole moment. On the right hand side the $1^{-}$excitation energy as well as the $B(E 1)$ and $B(E 3)$ transition probabilities (in Weisskopf units) are plotted as a function of mass number. The qualitative and even quantitative similarity with the $\mathrm{Cm}$ results presented in the previous section is striking and indicates that the addition of the two protons has little influence in the octupole deformation properties. Strong octupole correlation effects are predicted in the mass number range 228 - 236 and a severe quenching of $B(E 1)$ transition probability is anticipated for the nuclei around ${ }^{224} \mathrm{Cf}$. At this point it has to be mentioned that proton's drip line is predicted to be at ${ }^{228} \mathrm{Cf}$ in calculations with Gogny D1S [43. Therefore it is rather unlikely that the octupole effects expected in the range 228-236 will ever be accessible experimentally.

\subsection{Fermium and Nobelium}

For the Fermium $(\mathrm{Z}=100)$ and Nobelium $(\mathrm{Z}=102)$ isotopes calculations with neutron numbers in the range $\mathrm{N}=122$ and $\mathrm{N}=142$ have been carried out. Although octupole deformation is present in our calculations for the ground state of ${ }^{230-232} \mathrm{Fm}(N=$ $130-132)$ and ${ }^{232-234}$ No $(N=130-132)$ we have decided not to present the results 

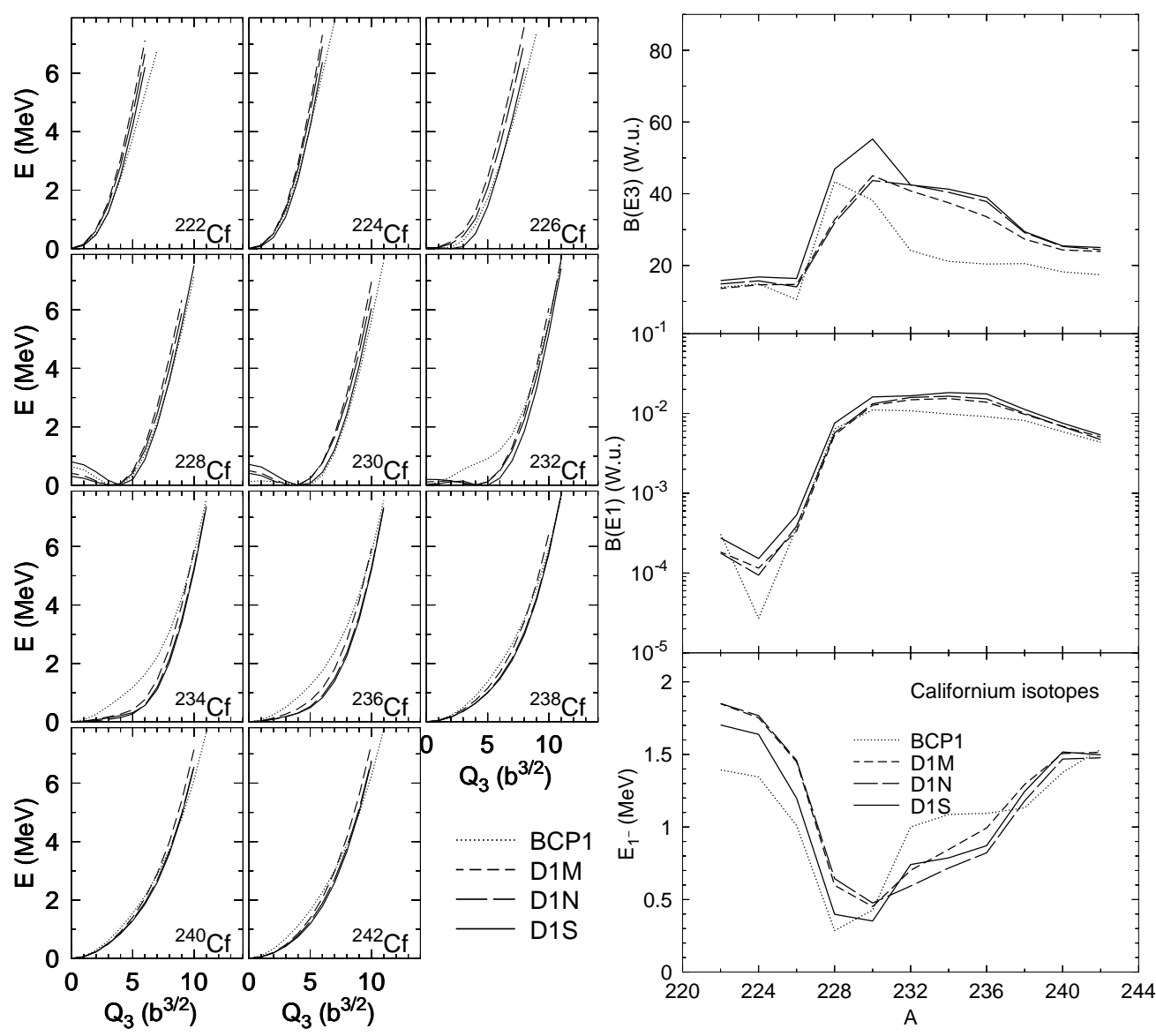

Figure 7. Same as figure 5 but for the californium isotopes

here as those nuclei are right at the proton's drip line, according to calculations with the Gogny D1S force (see [43] for details), and therefore it is rather unlikely that they will ever be created in the laboratory.

\section{Conclusions}

We have performed systematic mean field calculations for a relevant set of even-even actinides to explore the impact of octupole correlations in the low energy nuclear spectrum. We observe strong octupole correlations in some uranium, plutonium, curium and californium isotopes with a strength that decreases with proton number. The neutron number of the isotopes with strong octupole correlations become closer to the proton's drip line as proton number increases. As a consequence, the fermium and nobelium isotopes showing up noticeable octupole correlations are beyond the drip line. Excitation energies for the $1^{-}$state as low as $300 \mathrm{keV}$ and $B(E 3)$ transition probabilities of 60 to $70 \mathrm{~W} . u$. are predicted in some uranium isotopes, making them good candidates for experimental studies aiming to extend the region of known nuclei where octupole correlation effects are important. On the theoretical side, the 
similarity among the results obtained with three different parametrizations of the Gogny functional give credit to them as suited for an equivalent description of octupole correlation effects. The similitudes between the Gogny and BCP results indicate that the octupole correlation effects obtained are genuine and not an artifact of the phenomenological interactions used.

\section{Acknowledgments}

This work was supported by MICINN (Spain) under research grants FIS2008-01301, FPA2009-08958, and FIS2009-07277, as well as by Consolider-Ingenio 2010 Programs CPAN CSD2007-00042 and MULTIDARK CSD2009-00064.

[1] P.A. Butler and W. Nazarewicz, Rev. Mod. Phys. 66, 349 (1996)

[2] W.R. Phillips, I. Ahmad, H. Emling, R. Holzmann, R.V.F. Janssens, T.-L. Khoo and M.W. Drigert, Phys. Rev. Lett 57, 3257 (1986)

[3] E. Garrote, J.L.Egido and L.M. Robledo, Phys. Lett. B410, 86 (1997)

[4] E. Garrote, J.L. Egido and L.M. Robledo, Phys. Rev. Lett. 80, 4398 (1998)

[5] A. Bohr and B.R. Mottelson, Nuclear Structure (Benjamin, New York, 1969 and 1975)

[6] P. Ring and P. Schuck, The Nuclear Many Body Problem (Springer-Verlag Edt. Berlin, 1980)

[7] M. Bender, P.-H. Heenen and P.-G. Reinhard, Rev. Mod. Phys. 75, 121 (2003)

[8] J.L. Egido and L.M. Robledo, Nucl. Phys. A494, 85 (1989)

[9] J.L. Egido and L.M. Robledo, Nucl. Phys. A518, 475 (1990)

[10] J. Decharge and D. Gogny, Phys. Rev. C21, 1568 (1980)

[11] J.F. Berger, M. Girod and D. Gogny. Nucl. Phys. A428, 23c (1984)

[12] M. Baldo, P. Schuck, X. Viñas, Phys. Lett. B 663, 390 (2008)

[13] L. M. Robledo, M. Baldo, P. Schuck, and X. Viñas, Phys. Rev. C 77, 051301 (2008)

[14] L. M. Robledo, M. Baldo, P. Schuck, and X. Viñas, Phys. Rev. C 81, 034315 (2010)

[15] M. Baldo, L.M. Robledo, P. Schuck and X. Viñas. J. Phys. G37, 064015 (2010)

[16] L.M. Robledo, J.L.Egido, B. Nerlo-Pomorska and K. Pomorski, Phys. Lett. B201, 409 (1988)

[17] J. Skalski, P.-H. Heenen, P. Bonche, Nucl. Phys. A559, 221 (1993)

[18] K. Zberecki, P. Magierski, P.-H. Heenen, and N. Schunck, Phys. Rev. C 74, 051302 (2006)

[19] M. Yamagami, K. Matsuyanagi, M.Matsuo, Nucl. Phys. A693, 579 (2001)

[20] L.M Robledo and G.F. Bertsch, Phys Rev C84, 054302 (2011)

[21] L.M. Robledo and G.F. Bertsch, Phys. Rev. C84, 014312 (2011)

[22] D. Brink and W. Weiguny, Nucl. Phys. A120, 59 (1968)

[23] B. Giraud and B. Grammaticos, Nucl. Phys. A233, 373 (1974)

[24] P-G. Reinhard and K. Goeke, Rep. Prog. Phys. 50, 1 (1987)

[25] M. Baranger and M. Veneroni, Ann. of Phys. 114, 123 (1978)

[26] D.M. Brink, M.J. Giannoni and M. Veneroni, Nucl. Phys. A258, 237 (1976)

[27] F. Villars, Nucl. Phys. A285, 269 (1977)

[28] J.L. Egido and L.M. Robledo, Nucl. Phys. A524, 65 (1991)

[29] J.L. Egido, V. Martin, L.M. Robledo and Y.Sun, Phys. Rev. C53, 2855 (1996)

[30] B. Nerlo-Pomorska, K. Pomorski, M. Brack and E. Werner, Nucl. Phys. A462, 252 (1987)

[31] F. Villars, Nuclear Selfconsistent fields, Eds. G. Ripka and M. Porneuf (North Holland 1975)

[32] P.G. Reinhard and K. Goeke, J. of Phys. G4, 245 (1978)

[33] M. Girod and B. Grammaticos, Nucl. Phys. A330, 40 (1979)

[34] A. Baran, K. Pomorski, A. Lukasiak and A. Sobiczewski, Nucl. Phys. A361, 83 (1981)

[35] K. Boning, A. Sobiczewski, B. Nerlo-Pomorska and K. Pomorski, Phys. Lett. B161, 231 (1985).

[36] J.L. Egido and L.M. Robledo, Lecture Notes in Physics Vol 641, 269 (2004)

[37] F. Chappert, M. Girod, and S. Hilaire, Phys. Lett. B 668, 420 (2008)

[38] S. Goriely, S. Hilaire, M. Girod and S. Peru, Phys. Rev. Lett. 102, 242501 (2009)

[39] R. Rodriguez-Guzman, P. Sarriguren and L.M. Robledo, Phys. Rev. C82, 044318 (2010)

[40] R. Rodriguez-Guzman, P. Sarriguren, L.M. Robledo and S. Perez-Martin, Phys. Lett. B691, 202 (2010) 
[41] T. Kibédi and R. H. Spear, Atom. Data and Nucl. Data Tables, 80, 35 (2002)

[42] P. A. Butler and W. Nazarewicz, Nucl. Phys. A533, 249 (1991)

[43] S. Hilaire and M. Girod, Eur. Phys. J. A 33, 237 (2007) 